\title{
New Approach to Analysis of Noisy EELS Data
}

\author{
Emre Firlar ${ }^{1}$, Tanya Prozorov $^{1}$ \\ ${ }^{1}$ US DOE Ames Laboratory, Division of Materials Sciences and Engineering, Ames, IA USA
}

Electron Energy Loss Spectroscopy (EELS) is used to probe the chemical environment of materials at the nanometer scale. This analysis becomes challenging when dealing with embedded nanostructured materials, where small regions of interest residing in thick matrices yield noisy data. The data analysis can benefit from modeling the core loss spectra with the available reference peaks, using the experimental peak positions and ratio of the fitted peak areas to gauge the chemical binding in a material.

We applied this method for studying the early stages of magnetite biomineralization in magnetotactic bacteria. These microorganisms produce chains of magnetic nanocrystals via the uptake of soluble iron and iron oxide biomineralization in the intracellular membrane vesicles, called magnetosomes [1]. Even though this process has been studied for years, early stages of the magnetite magnetosome biomineralization pathway remains not fully understood [2].

Early stages of magnetosome magnetite biomineralization were investigated. To induce this process, iron-replete growth medium was added to the bacterial culture grown under the low iron conditions. We utilized Tecnai $G^{2}$ F20 Scanning Transmission Electron Microscope (STEM) equipped with Gatan Image Filter (GIF) to monitor the induced bacterial magnetosome formation in samples harvested at 30 min intervals. The data acquired from smallest $(\leq 10 \mathrm{~nm})$ nanocrystals was compared to those acquired on larger $(\geq 10 \mathrm{~nm})$ magnetosomes. A representative High Angle Annular Dark Field (HAADF)-STEM image from the bacterium with magnetosomes of the sizes ranging from 6 to $51 \mathrm{~nm}$ from a culture harvested 30 minutes after the induction, is shown in Figure 1A. From the electron diffraction analysis, the mature magnetosomes ( $45 \mathrm{~nm}$ ) were found to be fully crystalline magnetite (Figure 1B), while the electron diffraction pattern of the particles with sizes smaller than $10 \mathrm{~nm}$ (Figure 1C, D) is indicative of the presence of both crystalline magnetite and amorphous ferric oxyhydroxide (i.e. ferrihydrite $\left.\left(\mathrm{xFe} \mathrm{O}_{2} \cdot \mathrm{yH}_{2} \mathrm{O}\right)\right)$ structures.

After the initial characterization with the electron diffraction, core loss EELS was utilized to probe the oxidation state of iron in the biomineralized nanocrystals with varying sizes. Initial peak fitting of the EELS data was performed on a large magnetite magnetosome in the bacteria. Next, this approach was utilized to analyse smaller magnetosomes. Iron oxide particles have spectral signature around $709 \mathrm{eV}$ corresponding to the $\mathrm{Fe}_{3}$ edge. The EEL spectra acquired were modelled with $\mathrm{L}_{3}$ peaks of $\mathrm{Fe}^{2+}(707.8$ $\mathrm{eV}), \mathrm{Fe}^{3+}(709.5 \mathrm{eV})[3]$ and a satellite peak $(\sim 713.0 \mathrm{eV})$ [4]. From the EEL spectrum in Figure 2A, the roughly 2:1 ratio of the peak areas corresponding to $\mathrm{Fe}^{3+}$ and $\mathrm{Fe}^{2+}$, matches that expected for magnetite. Here the peak fitting was a straightforward process. On the other hand, the raw data acquired from a juvenile, poorly crystalline magnetosomes, were noisy, due to the small size of the particles residing in a thick bacterial cell, as shown in Figure 2B. To eliminate the ambiguity of the noisy spectrum, the data processed using the 5 and 20-point adjacent averaging smoothing methods were compared with the raw data. We have determined that excessive smoothing removes the small fingerprints, thus the averaging method should be evaluated in terms of both noise and the actual signal removal before the peak fitting is performed. Figure 2C and D show the peak fitting applied to the raw and processed data for the small 
magnetosome particle, respectively, using the previously determined peak positions. Based on the absence of $707.8 \mathrm{eV}$ peak attributable to the $\mathrm{Fe}^{2+}$, the $\mathrm{Fe}^{2+}$ concentration in small magnetosomes is below the detection limit. Furthermore, the peak position of the $\mathrm{L}_{3}$ was found to be shifted toward the higher energy in the smaller particles $(709.5 \mathrm{eV})$, consistent with the presence of only of $\mathrm{Fe}^{3+}$ in the smaller particles. EELS peak fitting and the electron diffraction data can be interpreted in terms of conversion of the as-formed ferric oxyhydroxide to magnetite occurring during the early stages of magnetosome formation and growth [5]. Further reduction of the ferric to ferrous iron during the crystallization and growth of these magnetosomes is also discussed. Current characterization methods include cryo-HAADF-STEM and in situ HAADF-STEM analysis.

This conventional TEM characterization in conjunction with this new approach to EELS fitting analysis proved to be very informative with regard to the structural analysis and interpretation of chemical environment of the bacterial magnetosome nanocrystals. We expect this method to be applicable for the data analysis of poorly crystalline nanomaterias located in thick matrices.

\section{References:}

[1] Woehl, Taylor J. et al, Scientific Reports 4: 6854, (2014)

[2] Bazylinski, Dennis A, Internatl Microbiol 2 (1999), 71-80

[3] Van Aken, P.A. et al, Phys Chem Minerals 25 (1998), 323-327

[4] De Groot, Frank M. F. et al, J. Phys. Chem. B 109 (2005), 20751-20762

[5] Baumgartner, Jens et al, Nature Materials 12 (2013), 310-314
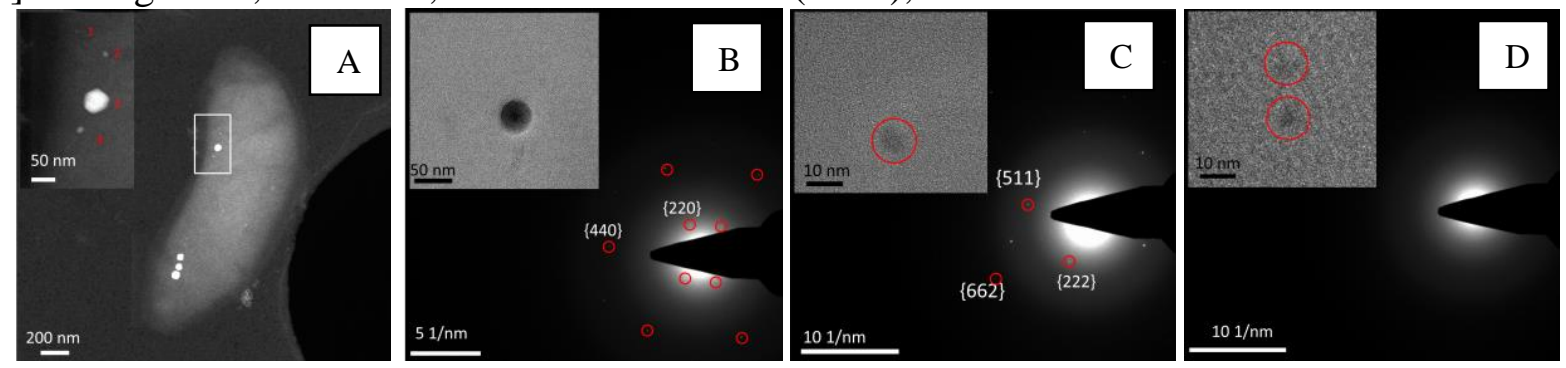

Figure 1. A) HAADF STEM image of 30 minute - time lapse sample. Inset shows (ref: white rectangle in low mag image) four different magnetosomes with sizes ranging from $6 \mathrm{~nm}$ to $51 \mathrm{~nm}$. Electron diffraction patterns and corresponding bright field TEM images of magnetosomes with sizes of B) 45 $\mathrm{nm}$ and C) $8 \mathrm{~nm}$ and D) $6 \mathrm{~nm}$ (2 particles).
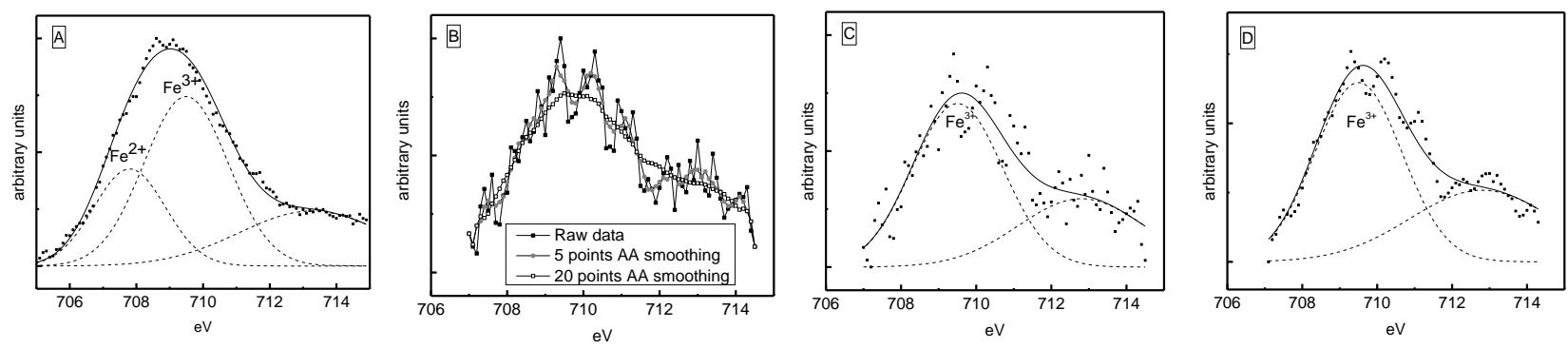

Figure 2. EELS Fe $\mathrm{L}_{3}$ core loss data from A) a big (size $48 \mathrm{~nm}$ ) and B) a small (size $10 \mathrm{~nm}$ ) magnetosome. A, C, D) Experimental data, fitting $\left(\mathrm{Fe}^{2+}: 707.8 \mathrm{eV}, \mathrm{Fe}^{3+}: 709.5 \mathrm{eV}\right.$ and a satellite peak around $713.0 \mathrm{eV}$ ) peaks and their sum are given by dots, dash lines and solid line, respectively. B) Raw data (solid line with black square) and smoothed data (5 points: solid line with grey sphere, 20 points: solid line with empty square) for the small magnetosome are compared. 